\title{
Dissociating perception from judgment in the action-specific effect of blocking ease on perceived speed
}

\author{
Jessica K. Witt ${ }^{1}$ • Nathan L. Tenhundfeld ${ }^{1}$ • Allison A. M. Bielak ${ }^{1}$
}

Published online: 14 October 2016

(C) The Psychonomic Society, Inc. 2016

\begin{abstract}
The action-specific approach to perception claims that a person's ability to act directly influences perceptual processes related to spatial vision. For example, a person's ability to block a moving ball impacts perceptual judgments of the ball's speed. However, an alternative explanation is that action rather than perception influences judgments. Here, we explore this distinction directly. Our method produces two distinct effects, one that is clearly a judgment-based effect and is based on the outcome of the trial (trial-outcome effect) and one that is under debate as to whether or not it is perceptual and is based on the ease with which the ball can be blocked (paddle-size effect). We explored whether these two effects would produce convergence or dissociations across various populations and manipulations. A dissociation is evidence for two separate underlying processes, whereas if the two effects did not dissociate, this would be consistent with claims that both effects were judgment-based. In Experiment 1 , we examined whether older and younger adults would show a dissociation between the two effects given some precedent for older adults to show greater susceptibility to nonperceptual factors in their judgments. In Experiment 2, we used a cover story to excuse poor performance and examined its effects on both types of effects. Both experiments revealed dissociations, suggesting that while one effect is judgment-based, the other effect is not. Coupled with prior research, we conclude that the action-specific effect of ease to block a ball on estimated ball speed is perceptual.
\end{abstract}

Jessica K. Witt

Jessica.Witt@ColoState.edu

1 Colorado State University, Fort Collins, CO 80523, USA
Keywords Perception and action $\cdot 3 \mathrm{D}$ perception: space perception · Aging

Vision scientists seek to understand both the sources of information for vision and the processes that operate on these sources. The action-specific account of perception argues that one source of information for vision comes from the motor system and is information about the perceiver's ability to perform the intended action (Witt, 2011, in press). Evidence for this claim comes from various sources. For example, softball players hitting better than others judge the ball as bigger, and those trained in parkour judge walls as shorter compared with novices (Gray, 2013; Taylor, Witt, \& Sugovic, 2011; Witt \& Proffitt, 2005). Hills appear steeper and distances appear farther to observers who are fatigued or overweight (Bhalla \& Proffitt, 1999; Sugovic, Turk, \& Witt, 2016; Taylor-Covill \& Eves, 2013, 2014, 2016). In addition, objects look closer and smaller when they are easier to reach and grasp (Linkenauger, Bulthoff, \& Mohler, 2015; Linkenauger, Leyrer, Buelthoff, \& Mohler, 2013; Witt, Proffitt, \& Epstein, 2005). These effects, known as action-specific effects, suggest that a person's ability to act influences spatial perception. The implication of these effects is that information about action should be considered a source of information for spatial vision.

The action-specific approach makes the bold claim that a person's ability to act directly influences spatial vision. But this claim has been challenged by the idea that responses, rather than perception, may have been altered. Vision cannot be measured directly and instead must be inferred based on behavioral responses. This leaves open the possibility that a given manipulation impacts the responses themselves rather than the underlying percept. There are many reasons why responses might be impacted, such as response bias or experimenter expectation (Durgin et al., 2009; Firestone, 2013; 
Firestone \& Scholl, 2014, in press-a; Philbeck \& Witt, 2015; Shaffer, McManama, Swank, \& Durgin, 2013; Wesp \& Gasper, 2012). Another reason is that participants may respond according to the felt difficulty of a given task or the affordance of the task rather than spatial perception of the target per se (Fajen \& Phillips, 2012; Woods, Philbeck, \& Danoff, 2009). In this case, they may truly believe their responses, but those responses would not reflect perception alone.

Recently, six pitfalls have been offered as ways to assess alternative explanations for purported top-down effects on perception (Firestone \& Scholl, in press-a). One of these pitfalls is to distinguish between perception and judgment. An action-specific effect could occur because the perceiver genuinely perceives the spatial layout of the environment differently, or because the participant alters her judgments despite seeing the environment in the same way. There are many reasons why one would alter her judgments. One reason relates to task demands or experimenter expectation. If the participant feels pressure to respond in a specific way (be it an external or an internal pressure), the participant can anticipate the experimenter's expectation and desires to be a good subject (Asch, 1955; Orne, 1962). Historically, these kinds of effects have been considered a subset of judgment-based effects, but recently they have been proposed to be their own, distinct pitfall, called response bias (Firestone \& Scholl, in press-a). An advantage of separating response bias from judgments into distinct categories is to emphasize that not all judgment-based effects are due to intentional modification of responses from the participant. Ruling out a response bias explanation does not necessarily rule out a judgment-based effect.

Distinguishing between perceptual effects and judgmentbased effects is of critical importance for theories of vision because genuine perceptual effects have implications for these theories whereas judgment-based effects do not. This debate between perception versus judgment can be seen within many different literatures, including the (purported) perception of causality and force (Hubbard, 2012, 2013; White, 2012), perception of animacy (Scholl \& Gao, 2013), categoryinfluenced perception of color (Levin \& Banaji, 2006), and motivation-, desire-, or fear-influenced perception of size, height, and distance (Balcetis \& Dunning, 2010; Cole, Balcetis, \& Dunning, 2013; Stefanucci \& Proffitt, 2009; Stefanucci, Proffitt, Clore, \& Parekh, 2008). In each case, evidence for a genuine perceptual effect, as opposed to an influence on judgments or other nonperceptual processes, has important implications for theories of vision. Similarly, if action's effect on perceptual judgments is due to underlying perceptual processes, the implication is that action is an additional source of information for spatial perception. Determining the underlying mechanism would require resolving both the content and form of the information related to action and establishing how this information exerts its effect on perceptual processes. In contrast, if action's effect on perceptual judgments ${ }^{1}$ is due to an effect on the judgments themselves, the findings could still be interesting, perhaps from a social psychology perspective, but would not have implications for theories of vision (except perhaps to suggest that current theories are correct to leave out information about a person's ability to act as an influence for spatial vision).

How can perception and judgment be distinguished? This issue has plagued perception research for a long time because there are always concerns as to whether a given phenomenon is perceptual, or, as mentioned above, due to judgments instead. The distinction cannot be made purely on the basis of the input because many theories of vision accept that certain kinds of nonoptical information can influence perception. Some of these kinds of influences have been described as unconscious inference (Helmholtz, 1925/2000), logical (Rock, 1983), intelligent (Gregory, 1997), or due to natural constraints or assumptions, such as the idea that light comes from above (Ramachandran, 1988), but are not considered to be judgment-based processes. As a result, the input cannot be used to determine if a given effect is perceptual. Instead, the distinction is between the visual experience (perception) and thoughts or beliefs about the target (judgment).

In some cases, the distinction between perception and judgment can be easily appreciated. For example, once a visual illusion has been explained, a perceiver can know that two objects are the same while still perceiving them as looking different (such as in the Ebbinghaus illusion). However, if explicit knowledge does not reveal a conflict between visual experience and physical realities, judgments might be based purely on what one sees. In these cases, it can be challenging to dissociate perception from judgment.

Several strategies exist to differentiate perception from judgment. Within the field of distance perception, the main strategy has been to examine convergence across measures, ideally with a variety of different kinds of measures, including direct and indirect as well as including judgments and actionbased measures (Foley, 1977; Gogel, 1990; Loomis \& Philbeck, 2008; Philbeck \& Loomis, 1997). The idea was that effects that revealed themselves across all measures were likely due to the underlying percept, whereas discrepancies across measures were instead indicative of cognitive correction, which is one type of judgment-based processes. Within the field of perception of causality and animacy, a strategy is to demonstrate a dependence of the phenomenon on subtle aspects of the visual stimulus (and independence from subtle aspects related to descriptions or knowledge of the stimulus) as evidence for a perceptual effect (Hubbard, 2013; Scholl \&

\footnotetext{
${ }^{1}$ The expression perceptual judgments was criticized by Firestone and Scholl (in press-a) for confusing this critical issue. However, the term honors the fact that often the distinction between perception and judgment has not yet been made without committing to either a perceptual or a judgment-based effect.
} 
Gao, 2013). Recently, Firestone and Scholl (in press-a) recommended another strategy, which was to define and eliminate the kinds of effects that are likely to lead to judgmentbased effects. For instance, if the experimenter were to call attention to a person's body weight, and then immediately ask the person to estimate distance to a target, this creates the kind of situation for which feelings about one's body size might alter one's judgments of distance. After eliminating these kinds of scenarios, if the action-specific effect were also eliminated, this would be evidence of a judgment-based effect. If the action-specific effect persisted, this would be evidence consistent with a perceptual explanation.

In this study we applied this logic to the action-specific effect of ease to block a ball on perceptual judgments of ball speed. In the task, known as the Pong method, a virtual ball moves across a computer screen at various speeds, and participants use a joystick to manipulate the position of a paddle to attempt to block the ball. We manipulate ease to block the ball by varying the size of the paddle on each attempt. After each attempt, participants estimate the speed of the ball. Typical results are that participants estimate the ball as moving faster when the paddle is small than when the paddle is big (Witt \& Sugovic, 2010, 2012). This difference in estimated speed between the big and small paddles will be referred to as the paddle effect.

The paddle effect has been examined using various methods to assess the perceptual nature of the effect. Many of these experiments can speak to the six pitfalls that have been raised by Firestone and Scholl (in press-a). For example, we have found that when visual differences between the paddle sizes are maintained, but performance-related differences are eliminated (such that ball blocking performance is not any better with the larger paddles), the differences in speed judgments are eliminated (Witt \& Sugovic, 2012; Witt, Sugovic, \& Taylor, 2012). This provides uniquely disconfirmatory findings (Pitfall 1) and rules out low-level differences (Pitfall 4). We have also conducted studies for which participants make their judgments while the ball is still moving and found similarly sized paddle effects, ruling out memory (Pitfall 6), as a potential explanation (Witt \& Sugovic, 2012, 2013a). In other studies, we controlled attention by using a dual task that forced participants to look at the ball and found that equating attention across the various conditions did not eliminate the paddle effect, thus ruling out attention (Pitfall 5) as an explanation (Witt, Sugovic, \& Dodd, 2016). These four pitfalls are the easiest to address, and studies have successfully examined, and subsequently ruled out, these pitfalls as explanations.

The remaining pitfalls (Pitfall 2: judgments; Pitfall 3: response bias) are more difficult to assess. Some work has been completed on both, but more work needs to be done. In one study, we attempted to measure participants' willingness to alter responses to comply with task demands (Witt \& Sugovic, 2013b). Participants were given instructions designed to bias their responses to be either faster or slower. Overall, participants who were given the "slow" instructions estimated the balls as moving slower compared with participants who were given the "fast" instructions, thereby showing some willingness to comply with the task demands set forth by the initial instructions. The research question was whether the paddle effect would emerge in participants who were less willing to alter their responses to comply with task demands. To measure compliance independent of the paddle effect, we performed a median split based on mean ball speed ratings. Those who rated the ball as slower were deemed more compliant if they had been given the "slow" instructions and less compliant if they had been given the "fast" instructions (and vice versa for participants who rated the ball as faster). As expected, the more compliant group showed the paddle effect. Critically, the less compliant group also showed the paddle effect, and its magnitude was the same extent as for the more compliant group. That participants who were less willing to alter their responses to comply with task demands still showed the paddle effect is evidence against a task demand explanation.

In other studies, we have used a variety of perceptual measures, including a speed bisection task (explained below), magnitude estimation (rate the speed on a scale of 1 to 7), and visual comparisons (indicate whether the ball is faster or slower than the comparison circle). All three types of measures demonstrated the paddle effect (Witt \& Sugovic, 2012) and convergence across measures points to a perceptual explanation over a judgment-based explanation (Loomis \& Philbeck, 2008). However, because there is no standard for ruling out judgment-based explanations, the goal of the researchers, more generally, is to address this critical issue using multiple strategies and multiple types of comparisons. These studies advance this literature by offering a novel way to apply current strategies to the Pong method.

In most studies on action-specific effects, there is a single manipulation of a person's ability to act, which produces a single effect in perceptual judgments. The goal is to then determine whether this effect is perceptual or falls prone to one of the six pitfalls. In contrast, the Pong method is unique because it leads to two co-occurring effects. The critical effect (by design) has been the effect related to the size (and effectiveness) of the paddle. We have argued that the paddle effect is perceptual (Witt, Sugovic, Tenhundfeld, \& King, in press), although we also acknowledge that more evidence is required to make this assertion.

A secondary effect relates to trial outcome. Trial outcome refers to whether the ball was successfully blocked or missed on each trial. Judgments of ball speed sometimes differ depending on trial outcome such that balls that are missed are estimated as moving faster compared with balls that are blocked. This difference in speed judgments as a function of blocking success will be referred to as the trial outcome effect. 
For the most part, the trial outcome effect has been neglected when analyzing data from the Pong method. Of the 21 studies published with this method, 14 were reported without any mention of trial outcome. There are two reasons that trial outcome has been neglected. One reason is difficulty in analyzing the data. The analysis of trial outcome does not lend itself to the same kind of analysis for the paddle effect. For the paddle effect, researchers can control the exact number of trials in each condition, thereby ensuring enough data to be able to calculate the point of subjective equality (PSE) for each paddle size. In contrast, researchers have less control over the number of trials that lead to hits versus misses as this differs across participants. In addition, paddle size and trial outcome are confounded. There are few misses with the big paddle, and approximately half of trials are missed with the small paddle. A second reason this factor has been neglected is that the few studies that did analyze trial outcome produced mixed outcomes. Of the seven experiments for which trial outcome was analyzed, four produced nonsignificant effects (Witt, South, \& Sugovic, 2014; Witt \& Sugovic, 2012), two were combined into a single analysis that produced a significant effect (Witt et al., 2016), and one produced a significant effect of trial outcome when participants played with the medium paddle but not with the small paddle (Witt \& Sugovic, 2010). Thus, the effect of trial outcome has not been particularly robust and thus has been frequently neglected in analysis.

However, we have regained interest in the trial outcome effect because trial outcome seems poised to create judgment-based effects in the Pong method. After a miss, a participant might infer that the miss occurred because the ball was moving fast, and judge the ball's speed accordingly. This pattern of responses would be analogous to that proposed by Wesp and Gasper (2012) regarding poor dart-dropping performance. We consider the trial outcome effect to be a judgmentbased effect because trial outcome is unknown until the end of the trial when the ball is either successfully blocked or missed, and thus seems unlikely to be able to influence perception of speed of the just-viewed ball. To argue that the trial outcome effect is perceptual would depend on one of two possibilities. There are small differences in the visual stimulation for a successful block (ball stops immediately) versus a miss (ball continues a short distance past the paddle). It could be possible that these small differences in visual information accounted for any differences in speed judgments due to trial outcome. Another possibility is that trial outcome could have postdictive effects on perceived ball speed. Trial outcome is unknown until the very end of the trial, so the only way that trial outcome could influence perception of the just-viewed ball is via postdictive processes. Perception of several events have been explained by appealing to postdiction, including the perception of the flash-lag effect (Eagleman \& Sejnowski, 2000), backward masking (Sheth, Nijhawan, \& Shimojo, 2000), and perception of causality (Choi \& Scholl, 2006).
However, in these cases, the event being judged occurs in a very short time interval (such as the moment the two objects come into contact with each other). It seems likely that brief events such as these would be more likely to be influenced by events that occur immediately afterwards (such as whether or not the two objects continue along their previous path) compared with tasks for which there is sustained information about the property to be judged (such as ball speed in the Pong method). Given that the trial outcome effect is not particularly robust, compared with these postdictive effects, and without any evidence to the contrary, we reject the idea that the trial outcome effect is perceptual. Note that any argument to the contrary would, in fact, support a perceptual interpretation of this particular action-specific effect.

Given that the effect of trial outcome is most likely a judgment-based effect, we can explicitly contrast the paddle effect with the trial outcome effect. If the two effects show converging patterns across various populations and manipulations, this would be evidence that the two effects are driven by a common underlying process. In this case, it would be evidence suggestive that both effects are due to postperceptual processes such as those involved in judgments. In contrast, if the paddle effect and the trial outcome effect showed a dissociation across populations or manipulations, this divergence would be evidence suggestive that the two effects are driven by separate processes. In this case, a dissociation would support the claim that the trial outcome effect is a judgment-based effect while the paddle effect is not a judgment-based effect.

\section{Experiment 1: Younger versus older adults}

In this experiment, we explored convergence versus divergence between the paddle effect and the trial outcome effect by comparing older and younger adults in the Pong method. Previous research with younger adults had shown that the effect of trial outcome was not particularly robust. However, we examined whether older adults might be more susceptible to adjusting their responses based on trial outcome. Relative to younger adults, older adults have shown increased susceptibility to biases in their perceptual judgments. For example, in one study, participants performed visual length discriminations for a series of lines (Norman et al., 2014). Both younger and older participants were biased by their previous responses: when a previous line had been labeled as "short," participants were more biased to respond that the current line was long. The susceptibility to this bias was stronger in older adults, who also showed the reverse bias to respond that the current line was short after having responded "long," a pattern not observed by the younger participants. Given that older adults were more likely to use irrelevant aspects of the task to inform their judgments of line length in that task, older adults might 
also be more likely to use the outcome of a Pong trial in our task to inform judgments of ball speed.

Other studies have shown judgment biases in older adults relative to younger adults. For example, when rating the likeability of vacation destinations of cities in the Midwest, both younger and older participants are more likely to decrease the likeability rating after rating extremely desirable vacation locations elsewhere (such as Honolulu, HI). If explicitly told the Midwestern cities were quite different than the very desirable locations, ratings did not decrease for the Midwestern cities for either group of participants. However, differences across the two groups emerged after the presentation of a subtle cue regarding the differences between the two groups of cities. Participants were told there were "more vacation spots to consider" Wang \& Chen (2004) (p. 537). With this subtle cue, judgments made by older adults were biased by the context and they rated the Midwestern cities as significantly worse than the extremely desirable cities, whereas this bias was not present in the judgments by younger adults (Wang \& Chen, 2004). Given that older adults were more biased in their ratings of the Midwestern cities by the context of the highly desirable locations, at least in the presence of a subtle cue, older adults might also be more biased in their judgments of ball speed given the context of a miss or a successful block in the Pong method.

The hypothesis in this experiment was that older adults, given their susceptibility to judgment-related biases due to prior responses or to context effects, would also be more susceptible to judgment-related biases in the Pong method and would thus show the trial outcome effect. If so, we can explore whether or not there is a dissociation between the two effects. If older adults show a trial outcome effect but not a paddle effect, this would be evidence that the paddle effect is judgment-based because it can be accounted for by another judgment-based effect. If older adults show both a trial outcome effect and a paddle effect, but younger adults show only a paddle effect, this would be evidence that the paddle effect is not due to judgment biases because the data would show a dissociation between the two processes.

\section{Method}

Participants Data from 43 participants living in Fort Collins, Colorado and surrounding areas were analyzed. The younger adult sample $(n=19)$ ranged from 18 to 37 years of age $(M=$ 20.39, $S D=4.39$; nine female, nine male, one unreported) and were all students attending Colorado State University. These participants were recruited via the online pool of available undergraduate participants. A power analysis showed that to obtain a between-subjects effect on the paddle effect such that the paddle effect was present for one group but not for the other required 42 participants, total (21 per group), to achieve $80 \%$ power. The effect size in the power analysis was Cohen's $d=.899$, which was based off of the data obtained in Experiment 3 by Witt et al. (2012).

The older adult participants were recruited as part of a larger study from the local senior center and community senior organizations via print and electronic advertisements. We aimed to get as many older adults as possible from this larger sample. The older adult sample $(n=24)$ was comprised of community-dwelling adults ages 60 to 84 years $(M=70.81$, $S D=6.12 ; 20$ female, four male). All older participants were able to see and hear clearly with corrective aids and scored 26 or greater on the Mini-Mental State Examination (Folstein, Folstein, \& McHugh, 1975). The older adults were highly educated $(M=16.71$ years, $S D=3.14)$. All rated their ability to see things up close as good to excellent, and the majority rated themselves as having good to excellent health $(n=$ $95.8 \%)$.

Stimuli and apparatus The experiment took place on a laptop with a $31.75 \mathrm{~cm}$ display (measured diagonally) placed on a table. The ball was a white circle that was $0.95 \mathrm{~cm}$ in diameter. The paddle was a white rectangle that was $0.13 \mathrm{~cm}$ in width and set to one of three heights $(0.90,2.95,5.95 \mathrm{~cm})$ on each trial. Participants controlled the vertical placement of the paddle using a joystick.

Procedure There were two training phases and one test phase. During the first training phase, participants were simply exposed to the slow and fast anchor speeds. Text on the screen indicated if the speed would be slow or fast, and then the ball moved from left to right with no vertical displacement at the slow $(13.5 \mathrm{~cm} / \mathrm{s})$ or fast $(54.1 \mathrm{~cm} / \mathrm{s})$ speed. Each speed was shown two times, and order was randomized. During the second training phase, no text was presented. The ball moved at the two anchor speeds, and after each presentation, participants indicated if the ball had moved at the slow speed or the fast speed by pressing the corresponding button on the joystick. Feedback was given after each response ("correct" or "incorrect" was shown on the screen). Performance during this training phase tends to be quite high. In this case, performance was $99 \%$ correct for older adults and $96 \%$ correct for younger adults. In cases for which a speed was misclassified, three of the four errors occurred on the first trial and thus were likely due to a misunderstanding of the task. During this phase, both speeds were shown two times, and order was randomized.

For the test trials, participants were told to try to block the ball and then estimate the speed of the ball as moving more like the slow speed or more like the fast speed. During each test trial, the paddle was set to one of three sizes, and the ball moved across the screen at one of six speeds ranging from 19 to $49 \mathrm{~cm} /$ s. The ball moved along a diagonal from left to right. The vertical direction (up vs. down) changed when the ball reached the top or bottom of the display and also at random. Each time 
the ball's position was advanced, there was a $5 \%$ chance of a change in vertical direction. Participants attempted to block the ball with the paddle by using the joystick. If the paddle was positioned correctly, the ball stopped on the paddle and the trial was coded as a successful block. If the paddle was not positioned correctly, the ball traveled past the paddle and the edge of the screen and the trial was coded as a miss. After each attempt, text was displayed on the screen that said "Slow or Fast?" and participants pressed the corresponding button on the joystick. A screen with the word "next" appeared for $500 \mathrm{~ms}$ before the start of the next trial. A block contained 36 trials ( 6 speeds $\times 3$ paddle sizes $\times 2$ repetitions). Order within block was randomized. Participants completed four blocks.

\section{Results}

Not surprisingly, paddle size had a substantial impact on ball blocking success (see Table 1). Proportion of balls successfully blocked was submitted to a repeated-measures ANOVA with paddle size as a within-subjects factor and age group as a between-subjects factor. Paddle size significantly influenced blocking success, $F(2,82)>545, p<.001, \eta_{\mathrm{p}}^{2}=.93$. Age group did not significantly affect blocking success, $F(1,41)=$ $0.33, p=.57, \eta_{\mathrm{p}}^{2}<.01$. The interaction between paddle size and age group was significant, with older adults showing increased effect of paddle size on proportion of balls blocked compared with younger adults, $F(2,82)=3.91, p=.026, \eta_{\mathrm{p}}{ }^{2}=.09$.

To assess the effect of paddle size on estimated speed, we initially analyzed the data by calculating the point of subjective equality (PSE) for each participant for each paddle size. The PSE is the standard calculation for these kinds of discrimination experiments (e.g., Gescheider, 1997) and corresponds to the speed that is experienced as exactly halfway between the slow and fast anchor speeds. A lower PSE corresponds with estimating the ball as moving faster (e.g., a slower speed is experienced as equal to the midpoint speed). PSEs were calculated by submitting the raw data (with "fast" responses coded as 1 and "slow" responses coded as 0 ) to separate logistic regressions for each participant and each paddle size. The result is that all the data are summarized as three PSEs per participant. Convergence was not achieved on eight of the 129 estimates. Lack of convergence was always due to quasicomplete separation. In these cases, SPSS's estimates of PSE are still useful and thus were included in the analysis.

Table 1 Proportion of balls successful blocked (and SEM) by age group and paddle size for Experiment 1

\begin{tabular}{lllll}
\hline Group & Small & Medium & Big & Mean \\
\hline Younger & $.39(.03)$ & $.69(.05)$ & $.84(.03)$ & $.64(.03)$ \\
Older & $.37(.03)$ & $.71(.04)$ & $.92(.03)$ & $.67(.03)$ \\
Mean & $.38(.02)$ & $.70(.03)$ & $.88(.02)$ & $.65(.02)$ \\
\hline
\end{tabular}

These PSEs were submitted to a repeated-measures ANOVA with paddle size as a within-subjects factor and age group as a between-subjects factor. Paddle size significantly influenced PSEs, $F(2,82)=44.28, p<.001, \eta_{\mathrm{p}}{ }^{2}=.52$. Age group did not significantly influence the PSEs, $F(1,41)=$ $0.10, p>.75, \eta_{\mathrm{p}}{ }^{2}<.01$. Interestingly, the interaction between paddle size and age group was significant, $F(2,82)=7.42, p=$ $.001, \eta_{\mathrm{p}}{ }^{2}=.15$ (see Fig. 1). These data suggest that older adults show a stronger action-specific effect than did younger adults. This can be contrasted with recent data showing that older adults do not show action-specific effects that relate to tool use (Costello et al., 2015). The Pong task could be considered akin to a tool-use task in that participants controlled a joystick and used the joystick to manipulate a virtual object that had the purpose of blocking the ball, and thus could be considered a virtual tool. Before interpreting the interaction between age group and paddle size, we next analyzed the data in a novel way for this method. As will be clear, the significant interaction confounded two separate effects, namely that of paddle size and that of trial outcome.

To look at the independent effects of paddle size and of trial outcome on estimated speed, researchers could calculate the PSE for each paddle size for each condition. However, there are not enough trials with the big paddle for which the ball was missed to permit calculation of a PSE in this condition. Thus,

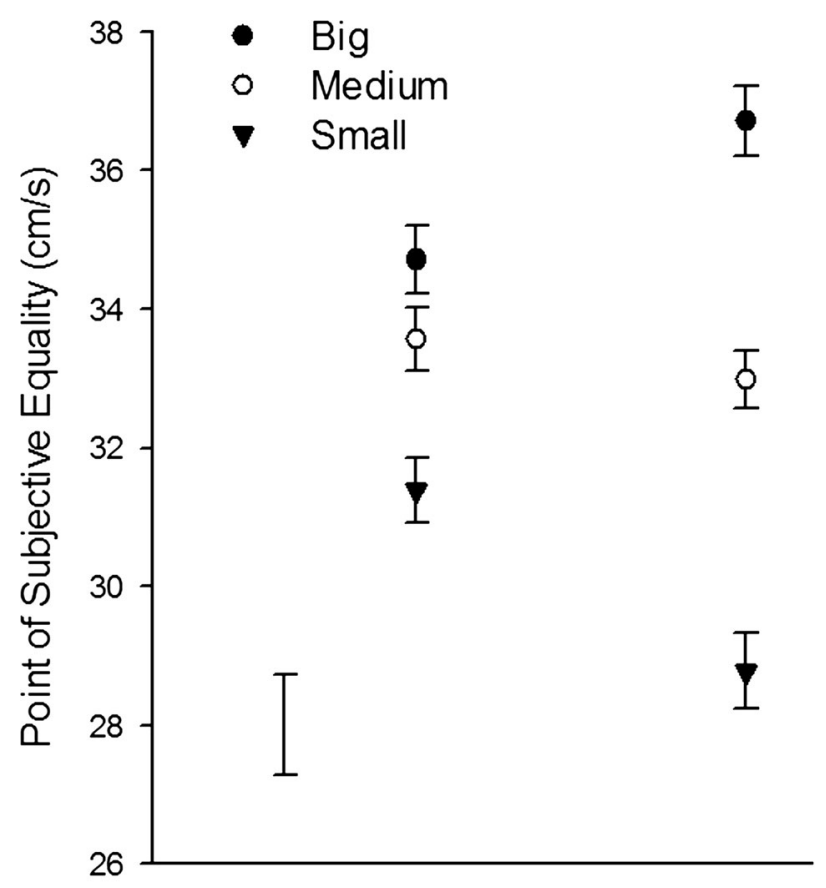

\section{Young Adults Older Adults}

Fig. 1 PSEs are plotted as a function of age group and paddle size for Experiment 1. A lower PSE corresponds with estimating the ball as moving faster. Error bars are 1 SEM calculated within-subjects for each age group. The single error bar in the bottom left corner represents the mean between-subjects SEM 
we instead analyzed our raw data by combining both factors in a single logistic regression for each participant. Trial response was entered as the dependent measure with same coding as before. Paddle size was coded as small $=-1$, medium $=0$, and big $=1$. Trial outcome was coded as a miss $=0$ and a successful block $=1$. Speed was entered as ball speed in $\mathrm{cm} / \mathrm{s}$. The outcomes of the logistic regressions were four coefficients for each participant (speed, paddle size, trial outcome, and a constant). We analyzed these coefficients as our dependent measures in subsequent analyses.

We first examined the coefficients for outliers. One older participant had a speed coefficient greater than 3 times the interquartile range, and one younger participant had a speed coefficient 1.5 times greater than the interquartile range. Another older participant had a paddle size coefficient less than 1.5 times the interquartile range. All three participants were excluded from the reported analyses, although inclusion of these participants did not change the statistical patterns or outcomes.

We ran a multivariate ANOVA with the coefficients for speed, paddle size, and trial outcome as dependent factors and age group as a between-subjects independent factor. The intercept for the coefficient for speed was significantly different than zero, $F(1,38)>395, p<.001, \eta_{\mathrm{p}}{ }^{2}=.91, M=.23, S E$ $=.01$. As ball speed increased, participants increased the proportion of "fast" responses. Age group significantly influenced the speed coefficient, $F(1,38)=9.10, p=.005, \eta_{\mathrm{p}}{ }^{2}=$ .19 . Older adults $(M=.20, S E=.016)$ were less sensitive to ball speed compared with younger adults $(M=.27, S E=.017)$.

The coefficient for paddle size was significantly different than zero, $F(1,38)=57.51, p<.001, \eta_{\mathrm{p}}{ }^{2}=.60, M=-.44, S E=$ .06 . This result shows that paddle size impacted perceptual judgments such that participants estimated the ball as moving faster as paddle size decreased. This replicates the typical paddle effect. Age group did not significantly influence the paddle size coefficient, $F(1,38)=0.01, p>.92, \eta_{\mathrm{p}}^{2}<.01$. Thus, the interaction that we saw when analyzing the PSEs did not replicate when we analyzed the coefficients. This is likely because the original calculation of PSEs for each paddle size included the confound of trial outcome, and this confound was not included in the calculation of these paddle size coefficients. Both younger $(M=-.45, S E=.09)$ and older $(M=$ $-.44, S E=.08)$ showed significant effects of paddle size, onesample $t \mathrm{~s}<-4.17, p \mathrm{~s}<.001$ (see Fig. 2).

The coefficient for trial outcome was significantly different than zero, $F(1,38)=7.45, p=.01, \eta_{\mathrm{p}}{ }^{2}=.16, M=-.34, S E=$ .12. Participants estimated the ball as moving faster after they missed than after they successfully caught the ball. Of interest, age group significantly influenced the coefficient for trial outcome, $F(1,38)=7.24, p=.01, \eta_{\mathrm{p}}{ }^{2}=.16$. Older participants $(M$ $=-.67, S E=.17)$ showed a greater effect of trial outcome compared with younger participants $(M=-.005, S E=.18)$. We submitted the trial outcome coefficients to separate onesample $t$ tests for each age group. For older participants, this coefficient was significantly less than zero, $t(21)=-4.04, p=$ $.001, d=.80$. For younger adults, this coefficient was not significantly different than zero, $t(17)=-0.03, p=.98$.

That older adults showed the effect of trial outcome, whereas younger adults did not, suggests that older adults are more susceptible than younger adults to judgment-related biases in this task. This finding is consistent with literature showing older adults are more susceptible to other kinds of biases such as biases due to previous judgments of line length (Norman et al., 2014) or biases due to context effects (Wang \& Chen, 2004). Increased susceptibility in older adults to bias their judgments is also apparent in other literatures such as false memories (Jacoby \& Rhodes, 2006) and increased sensitivity to age-related stereotype threats (Hess, Auman, Colcombe, \& Rahhal, 2003). Just as older adults have shown increased susceptibility to these biases previously, we found that they also were more likely to bias their judgments of ball speed based on whether or not they successfully blocked the ball.

\section{Discussion}

The key theoretical question was whether postperceptual processes such as those involved in generating judgments or those related to response bias can account for the paddle effect. For example, after missing the ball, participants might infer that they missed because the ball was moving faster. These inferences could lead to the paddle effect because participants miss more frequently with the small paddle, and thus would judge the ball as moving "fast" more often, than with the big paddle. The first piece of evidence against this explanation is that the differences in estimated speed across paddle size persisted even when taking into account the outcome of the trial in terms of whether the ball was missed or successfully blocked. In other words, even after accounting for the scenario that is likely to lead to postperceptual inferences about ball speed, the paddle effect still emerged.

The second piece of evidence against a postperceptual explanation is the dissociation that emerged across the age groups. Age group impacted the effect of trial outcome on speed judgments but not the effect of paddle size on speed judgments. This divergence in effects across populations is consistent with the proposal that separate processes underlie the effects of trial outcome and paddle size. In the case of trial outcome, the most likely process involves postperceptual inference about ball speed. The theoretical implication is that another process, one not involving postperceptual inference, underlies the paddle effect.

\section{Experiment 2: Cover stories}

We wanted to explore the dissociation between the two effects (paddle size and trial outcome) in a different way. Specifically, 


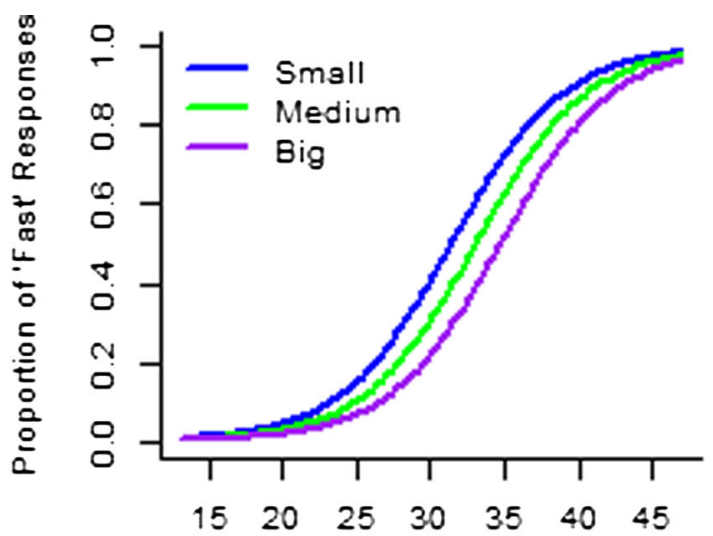

Ball speed $\{\mathrm{cm} / \mathrm{s}$ )

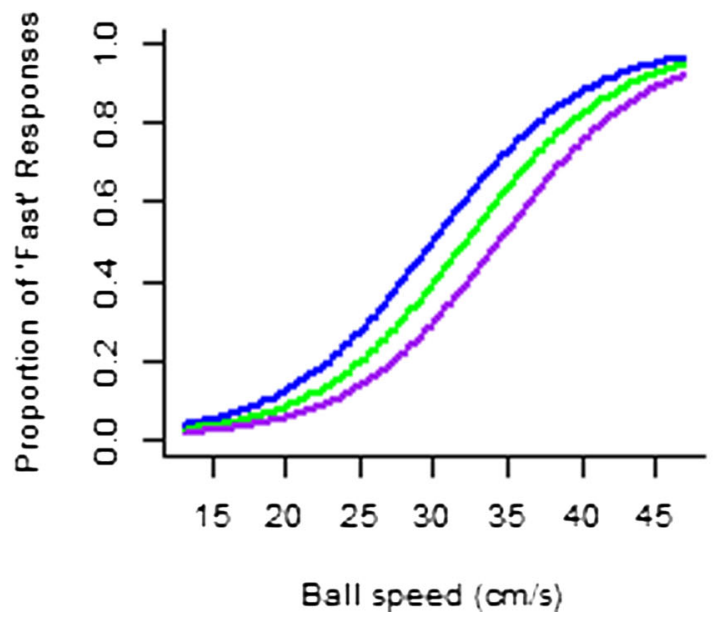

Fig. 2 Logistic regression curves based on mean coefficients for younger (top row) and older (bottom row) adults for Experiment 1. Left column shows main effect of paddle size (keeping trial outcome constant), and

we wanted to examine whether divergence between the two effects would also be apparent for experimental manipulations in addition to divergence across populations of participants. In describing the pitfall related to judgments, Firestone and Scholl (in press-a) offered a dart-dropping experiment as a case study of how the effect of performance on judged size could be due to people adjusting their responses. In the study, participants attempted to drop darts onto a circular target positioned on the floor. They were given up to 15 tries, stopping as soon as they successfully hit the target or after all 15 attempts, at which point they estimated the size of the target. Previously, it was found that those who hit the target with fewer attempts judged the target to be bigger (Wesp, Cichello, Gracia, \& Davis, 2004). To examine a possible role for judgments, rather than genuine differences in perceived size, Wesp and Gasper (2012) ran a second study in which they divided participants into two groups. The excuse group was told the darts were of "really bad quality" that was making it difficult for people to hit the target. The no-excuse group was told nothing about the darts, thus replicating their original study design. The thinking was as follows: After
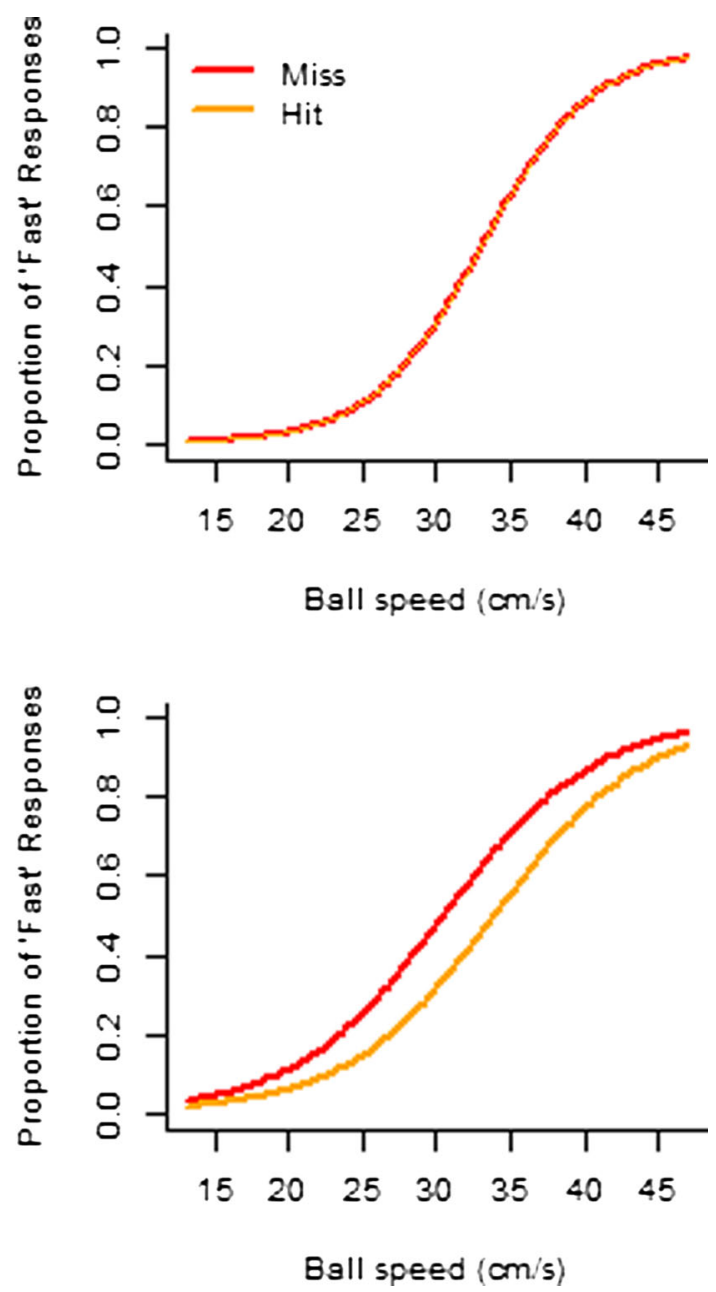

right column shows main effect of trial outcome (keeping paddle size constant). Two lines are plotted in the top-right graph but are difficult to differentiate because of their overlap

requiring more attempts to hit the target, participants might try to justify their poor performance by judging the target as smaller, but for participants who could blame the darts rather than their own performance, such justification was unnecessary. In line with this reasoning, participants in the no-excuse group showed a significant correlation between dart dropping performance and estimated size, but the correlation was much weaker and not significant for participants in the excuse group.

Applying this logic to the Pong method, participants might be more inclined to infer the ball was moving fast after a miss than after a hit. However, if a cover story provided participants with an external reason for poor performance, such that the task was especially challenging because the ball moved randomly, participants would be less likely to make inferences of ball speed based on their performance. If a cover story that provided an external reason for why a task would seem difficult eliminated the paddle effect, this would be evidence consistent with a judgment-based explanation. If the paddle effect persisted despite such a cover story, the result would be evidence against a judgment-based explanation. 


\section{Method}

Participants Sixty-four students at Colorado State University volunteered in exchange for course credit. Data from the first 36 participants have been previously reported in a footnote (Witt \& Sugovic, 2013b). Additional participants were run to achieve $90 \%$ power. Given that a null effect would have critical theoretical implications, we wanted to ensure that any null effects would not be due to insufficient statistical power.

Stimuli and apparatus Stimuli were presented on a 19-in. desktop display with a black background. The stimuli consisted of a white circle, $1.6 \mathrm{~cm}$ in diameter, which served as the ball. During training, the ball moved at a slow $(18 \mathrm{~cm} / \mathrm{s})$ or a fast $(74 \mathrm{~cm} / \mathrm{s})$ speed, and it moved horizontally from left to right with no vertical displacement. During test trials, the ball moved at one of six speeds ranging from 26 to $67 \mathrm{~cm} / \mathrm{s}$. The ball moved from left to right and along a diagonal, changing directions at the bottom and top of the display and also at random.

A white rectangle with a black outline served as the paddle. It was $0.8 \mathrm{~cm}$ wide and set to one of three heights $(1.9,5.8$, or $11.8 \mathrm{~cm}$ ) on each trial. Participants used a joystick to control the vertical position of the paddle. The two buttons on the front of the joystick were labeled "slow" and "fast" and used to make speed judgments. The paddle was placed on top of another white rectangle that was the same width as the paddle and the same height as the display (see Fig. 3). As a result, the visual information that specified the height of the paddle was the two black lines from its outline. This set-up eliminated luminance differences across the three paddle sizes, which are likely irrelevant, but we tried to minimize any differences in the visual information. The background rectangle had no impact on the ball's movements, and the ball simply passed over the rectangle in cases for which the ball was missed.

Procedure Participants were first exposed to the slow and fast anchor speeds. Text on the screen indicated which speed would be shown for each exposure. Each speed was shown three times, and order was randomized. Then participants

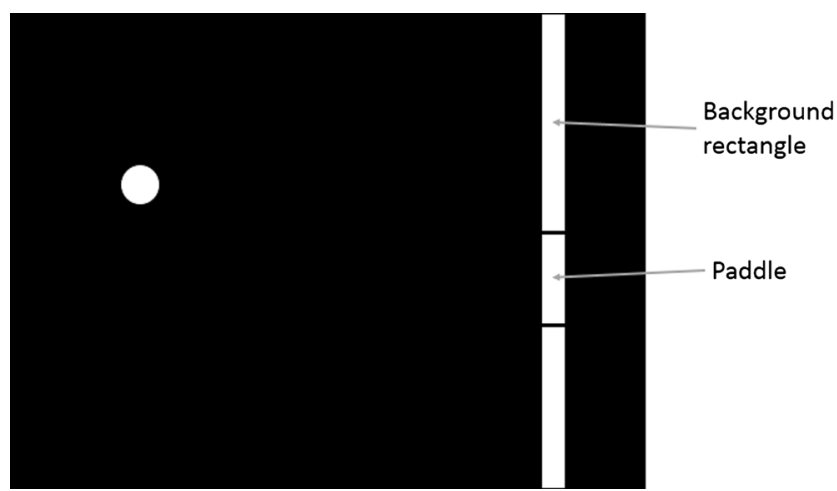

Fig. 3 Illustration of a sample display were tested on their ability to discriminate the two anchor speeds. During this phase, the speed was presented without the accompanying text, and participants had to indicate if it was the slow speed or the fast speed by pressing the corresponding button on the joystick. Feedback was given after each response, and performance was at $99 \%$ correct. Again, each speed was presented three times, and order was randomized.

After training, participants received instructions on how to perform the main task. Both groups were told to block the ball by correctly positioning the paddle. Then the easy group was told that the task would be easy, and the hard group was told that the task would be hard (see Table 2). Finally, participants were told to block as many balls as they could and for each, indicate whether the ball moved more like the fast speed or more like the slow speed.

After instructions, the rest of the experiment was exactly the same for both groups. For each test trial, the ball moved at one of six speeds, and the paddle was set to one of three heights. If the paddle was positioned correctly, then the ball stopped on the paddle and it was scored a successful block, otherwise the ball continued beyond the edge of the screen and the trial was scored a miss. At this point, text appeared on the screen displaying "Slow or Fast?" and participants made their perceptual judgments by pressing the corresponding button on the joystick. A $500 \mathrm{~ms}$ screen with the word "next" appeared before the start of the next trial. One block consisted of 36 trials in which all six test speeds were shown twice with each paddle size, and order was randomized. Participants completed six blocks for a total of 216 trials.

\section{Results}

Ball blocking success is summarized in Table 3. Proportion of balls successful blocked were submitted to a repeatedmeasures ANOVA with paddle size as a within-subjects factor and cover story condition as a between-subjects factor. Paddle size significantly influenced blocking success, $F(2,124)>$ 1,522. Cover story condition did not significantly influence blocking success, $F(1,62)=0.70, p>.40$. The interaction between paddle size and cover story was not statistically significant, $F(2,124)=2.60, p=.079, \eta_{\mathrm{p}}^{2}=.04$.

Table 2 Unique instructions given to each cover story condition

\begin{tabular}{ll}
\hline Condition & Instruction \\
\hline Easy & $\begin{array}{c}\text { For the first part of this experiment, you will find the } \\
\text { task easy because the movements of the paddle } \\
\text { will perfectly match the movements of the joystick. } \\
\text { For the first part of this experiment, you will find the } \\
\text { task to be more difficult because the ball will } \\
\text { change directions randomly. }\end{array}$ \\
\hline
\end{tabular}


Table 3 Proportion of balls successful blocked (and SEM) by cover story condition and paddle size for Experiment 2

\begin{tabular}{lllll}
\hline Cover Story & Small & Medium & Big & Mean \\
\hline Easy & $.46(.01)$ & $.81(.02)$ & $.95(.01)$ & $.74(.01)$ \\
Hard & $.47(.01)$ & $.79(.02)$ & $.93(.01)$ & $.73(.01)$ \\
Mean & $.47(.01)$ & $.80(.01)$ & $.94(.01)$ & $.74(.01)$ \\
\hline
\end{tabular}

The raw ball speed estimates were submitted to separate logistic regressions for each participant with ball speed (in $\mathrm{cm} /$ s), paddle size $(-1,0,1)$, and trial outcome $(0,1)$ as predictors. The data were first explored for outliers. Several participants had one coefficient between 1.5 and 3 times the interquartile range. Analyses were conducted with and without these participants. Similar patterns emerged with significant $p$ values being somewhat lower with outlier removal, as would be expected. To be more conservative, we decided to report analyses with all of the data included. Coefficients for speed, paddle size, and trial outcome were submitted to a multivariate ANOVA with cover story condition as the predictor. The intercepts for all coefficients were significant, all $p \mathrm{~s}<.001$. The main effect for cover story was not significant for any coefficient, $p \mathrm{~s}=.28-.36, \eta_{\mathrm{p}}{ }^{2}=.01-.02$. This suggests that our cover story was ineffective for creating, or eliminating, response biases. It is unclear as to what makes some cover stories effective and other ineffective.

However, we observed that the variance for the coefficient for trial outcome (.50) was nearly 4 times greater than the variance for the coefficient for paddle size (.13). This made us question whether other factors contributed to the trial outcome coefficient. Given that the cover story is supposed to provide an excuse for poor performance (Wesp \& Gasper, 2012), it made sense to look at ball blocking performance as a potential factor for when the cover story might be effective. We performed a median split on mean number of balls successfully blocked for each cover story condition ( $n=16$ for each group), and included this as a factor in the MANOVA. The coefficients for speed, paddle size, and trial outcome were dependent factors, and ball blocking performance group and cover story condition were between-subjects factors.

The intercept for the coefficient for speed was significant, $F(1,60)>698, p<.001, \eta_{\mathrm{p}}{ }^{2}=.92, M=.198, S E=.007$, showing that participants were sensitive to the speed of the ball. Cover story condition did not significantly influence the speed coefficient, $F(1,60)=1.35, p=.25, \eta_{\mathrm{p}}{ }^{2}=.02$, suggesting no difference in sensitivity to ball speed across the two groups of participants (easy: $M=.19, S E=.01$; hard: $M=.21$, $S E=.01)$. Blocking performance group significantly influenced the speed coefficient, $F(1,60)=12.88, p=.001, \eta_{\mathrm{p}}{ }^{2}$ $=.18$. Participants who blocked less successfully also exhibited reduced sensitivity to ball speed $(M=.17, S E=.01)$ compared with participants who blocked more successfully
$(M=.22, S E=.01)$. A higher speed coefficient indicates a greater change in responses as ball speed increases, so higher values correspond to increased sensitivity to ball speed. In this case, a likely explanation is that those who perceived ball speed better had more success blocking the ball. There was no significant interaction between cover story condition and performance group on the speed coefficient, $F(1,60)<.04, p$ $>.85, \eta_{\mathrm{p}}^{2}<.01$.

The intercept for the coefficient for trial outcome was also significant, $F(1,60)=19.41, p<.001, \eta_{\mathrm{p}}{ }^{2}=.24, M=-.378$, $S E=.086$. The negative coefficient indicates that the ball was judged as moving faster after a miss compared with a successful block. In contrast to Experiment 1, the young participants in this sample showed a significant effect of trial outcome. As stated earlier, younger participants have shown this effect in the past, but it is not robust and only seems to be significant approximately $25 \%$ of the time. One possibility is that this experiment just happened to fall in the $25 \%$ of experiments for which an effect of trial outcome was observed. Another possibility is that the significant effect in this case may relate to the cover stories, which clearly highlighted ball blocking performance in both conditions. In contrast, in Experiment 1, participants were told to try to block the ball, but this aspect of the task was not discussed beyond this initial, brief instruction. Perhaps emphasizing this aspect as being important during the instructions is more likely to lead to an effect of trial outcome. This would be consistent with the idea that cover stories can produce their own response biases. In future research, this speculation needs to be tested directly, however, to more fully understand the link between cover stories and task demands.

Neither cover story condition nor ball blocking performance group significantly influenced the coefficient for trial outcome, $F_{\mathrm{S}}<1$. However, the interaction between cover story condition and performance group was significant, $F(1$, $60)=5.43, p=.023, \eta_{\mathrm{p}}{ }^{2}=.08$ (see Fig. 4). The cover story

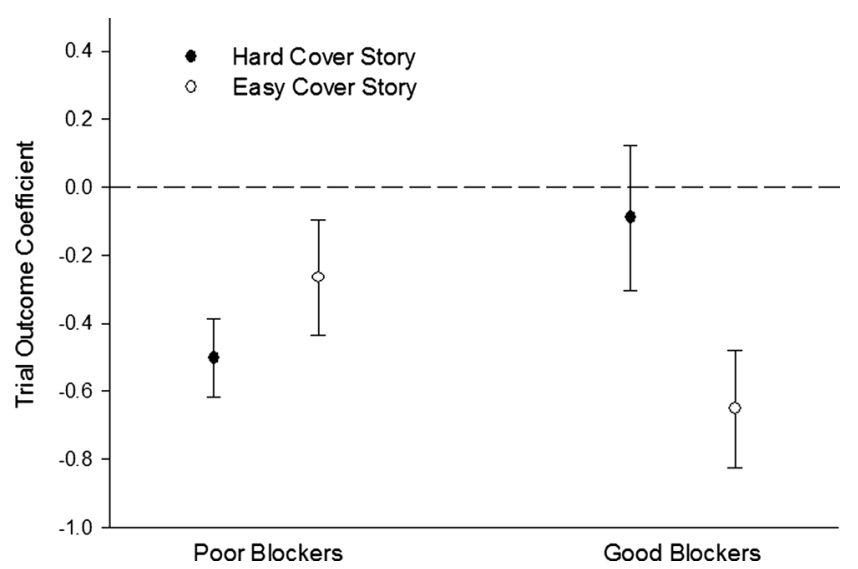

Fig. 4 Mean coefficients for trial outcome plotted as a function of blocking performance and cover story for Experiment 2. A greater negative value indicates a larger effect of trial outcome on speed judgments. The dashed line indicates no effect of trial outcome. Error bars are 1 SEM calculated between subjects 
was effective at altering the influence of trial outcome on judgments for participants who were better at blocking the ball. This was confirmed by an independent-samples $t$ test for the better blockers across the two cover story conditions, $t(30)=2.04, p=.051$, Cohen's $d=0.72 .^{2}$ For the better blockers, those who were given a cover story that helped excuse their poor performance showed no effect of trial outcome, $t(15)=-0.42, p=.68, d=0.10$. In contrast, for better blockers, those given a cover story that provided no excuse showed a strong influence of trial outcome on judgments, $t(15)=-3.76, p=.002, d=0.94$. For worse blockers, cover story did not impact the influence of trial outcome on judgments, $t(30)=-1.17, p=.25, d=0.42$.

The intercept for the coefficient for paddle size was significant, $F(1,60)=89.21, p<.001, \eta_{\mathrm{p}}{ }^{2}=.60, M=-.423, S E=$ .045. Perceptual judgments of ball speed decreased as paddle size increased, showing the typical effect of paddle size on estimated ball speed. The critical question was whether the cover story provided by the instructions impacted the effect of paddle size on estimated ball speed. The main effect of cover story condition did not significantly influence the coefficient for paddle size, $F(1,60)=0.97, p=.33, \eta_{\mathrm{p}}{ }^{2}=.02$, and the interaction between condition and ball blocking performance group did not reach significance, $F(1,60)<0.01, p>$ $.99, \eta_{\mathrm{p}}{ }^{2}<.01$ (see Fig. 5). In contrast to trial outcome, the coefficient for paddle size was not influenced by the cover story and its provision of an excuse for poor performance. Ball blocking performance group also did not significantly influence the coefficient for paddle size, $F(1,60)=0.44, p>$ $.51, \eta_{\mathrm{p}}{ }^{2}=.01$.

\section{Discussion}

The cover story was sufficient to eliminate the effect of trial outcome on estimated speed, at least for the good blockers. We did not expect, a priori, that better blockers would be more influenced by the cover story than good blockers. The data from Wesp and Gasper (2012) was not described in sufficient detail to be able to assess whether their cover story had a greater impact on those with better or worse dart performance. It is reasonable that a simple cover story would impact participants in different ways. Indeed, even after accounting for ball blocking performance, the variance for the coefficient for trial

\footnotetext{
${ }^{2}$ Given that 4 of the 6 outliers were identified based on their coefficient for trial outcome, this is the one statistical result for which removing outliers had a notable impact on the $p$ value. Without these outliers, the interaction between cover story condition and performance group was significant, $F(1,54)=6.59, p=.013, \eta_{\mathrm{p}}^{2}=.11$. For the better blockers, there was a significant effect on the coefficient for trial outcome based on cover story condition, $t(26)=2.82, p=.009, d=1.07$ (hard cover story: $M$ $=.06, S E=.15$; easy cover story: $M=-.56, S E=.16$ ). For the worse blockers, the difference between cover story conditions was not significant, $t(28)=-0.73, p=.47, d=.27$ (hard cover story: $M=-.27, S E=.17$; easy cover story: $M=-.41, S E=.10)$.
}

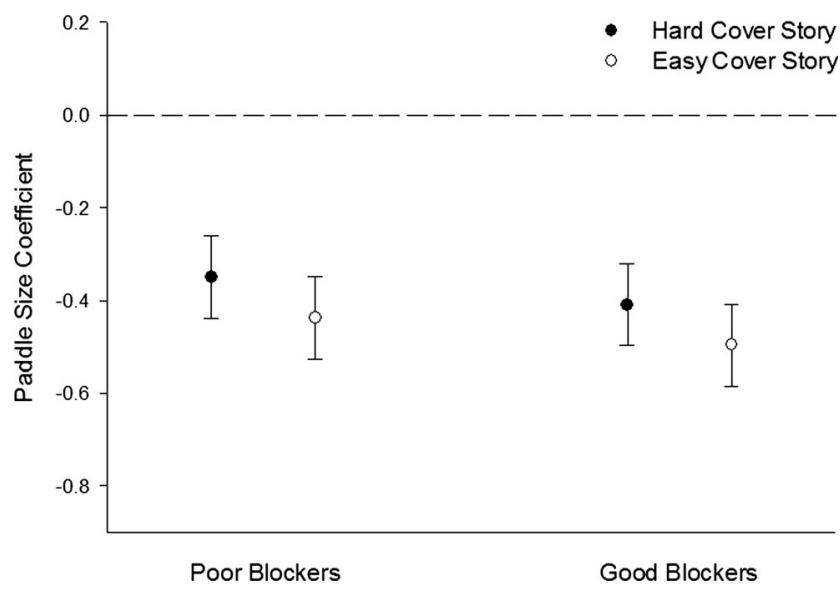

Fig. 5 Mean coefficients for paddle size plotted as a function of blocking performance and cover story for Experiment 2. A greater negative value indicates a larger effect of paddle size on perceptual speed judgments. The dashed line indicates no effect of paddle size. Error bars are $1 S E M$ calculated between-subjects

outcome was still more than 3 times greater than the coefficient for paddle size, suggesting a large range of individual differences for the judgment-based effect of trial outcome. To more fully understand the complex and nuanced ways in which cover stories might alter judgment-based effects, more research should be conducted on the relationship between cover stories, performance, and judgments.

The key experimental question was whether a cover story that would explain away poor performance would also reduce the effect of paddle size on estimated speed. In contrast to the findings with darts (Wesp \& Gasper, 2012), we did not find any reduction due to the cover story for our main actionspecific effect. Cover stories such as the one we used were argued to be an effective method for testing whether a given effect is driven by judgments rather than perception (Firestone \& Scholl, in press-a). That the paddle effect emerged even in spite of the cover story is evidence against a judgment-based interpretation.

\section{General discussion}

What are the sources of information for spatial vision? Obviously, the most influential source of information comes from optical cues, but they do not constitute the only source of information. Some information comes from oculomotor cues. Other sources of information include innate, natural, or learned constraints, sometimes known as priors, such as the assumption that light comes from above (Ramachandran, 1988), that objects tend to be about $2 \mathrm{~m}$ away (Gogel, 1990), or that objects tend to be stationary (Stocker \& Simoncelli, 2006). The cross-modal literature has demonstrated that information from other senses such as audition can also influence vision (Sekuler, Sekuler, \& Lau, 1997; Shams, 
Kamitani, \& Shimojo, 2000). Relatedly, information from haptic experience can influence visual perception of force and causality (White, 2012). The action-specific approach proposes another source of information for spatial vision, namely information about a person's ability to perform the intended action (Witt, 2011, in press). However, with each proposal for a new source of information for spatial vision comes much skepticism and controversy, and the actionspecific approach is no different. Researchers within this approach have drawn insight on how to empirically determine whether or not an effect is perceptual from historic and ongoing debates related to these issues across the various literatures including cross-modal effects, influences of priors, and other purported top-down effects on perception.

The central research question in this study was to explore convergence versus dissociation across two simultaneous effects within the Pong method. The critical effect is of paddle size, which has been hypothesized to be a perceptual effect such that perceivers genuinely see the ball's movement as faster when they play with the small paddle compared with the big paddle (Witt \& Sugovic, 2012, 2013a, 2013b; Witt et al., in press). The second effect is that of trial outcome, which refers to whether or not the ball was blocked successfully or missed on each trial. Trial outcome is assumed to be a judgment-based effect given that the outcome occurs after or at the end of the visual information that specifies ball speed.

If the paddle effect produces similar patterns as the trial outcome effect across populations and manipulations, this would suggest the two effects are driven by the same underlying processes, namely judgments. In contrast, if the paddle effect produces a different pattern of results compared with the trial outcome effect, this would suggest different underlying processes, namely that the judgment-based processes driving the effect of trial outcome were not giving rise to the paddle effect. This would be evidence against a judgment-based explanation of the paddle effect.

In Experiment 1, we compared both effects across two different populations: younger and older adults. Older adults were selected because of prior evidence demonstrating increased susceptibility to biases in their responses based external (nonperceptual) factors (e.g., Norman et al., 2014). Consistent with the idea that older adults are more susceptible to external biases, we found that older, but not younger, adults exhibited biases in their speed judgments that were due to trial outcome (whether or not they successfully blocked the ball on each trial). In contrast, the paddle effect was consistent for both groups of participants. This reveals a divergence between the two effects across different populations.

In Experiment 2, we explored whether the paddle effect and the trial outcome effect would converge or dissociate across an experimental manipulation. Inspiration came from a specific proposal by Wesp and Gasper (2012), which was strongly endorsed by Firestone and Scholl (in press-a), that a critical way to differentiate perception from judgment was to deliver a cover story that provided a reason for a person's poor performance. The goal of this kind of cover story is that participants will not feel the need to believe that the target was smaller to account for poor performance. By eliminating this pressure, researchers can explore whether any difference in judgments is also eliminated, in which case, the implication is that the effect is due to judgments, not perception. As predicted, the cover story impacted the influence of trial outcome on speed judgments, although this effect was mediated by ball blocking performance. The cover story that excused poor performance successfully eliminated the effect of trial outcome for participants who were better blockers. In contrast, the effect of trial outcome was apparent for all other conditions. This confirms, at least to some extent, the effectiveness of the cover story, and permits the critical assessment of whether the cover story also impacted the effect of paddle size on estimated speed. Participants showed a significant effect of paddle size on estimated speed, and neither the cover story nor its interaction with ball blocking performance modulated the extent of this effect. Given the claim that an effect of cover story proves the effect is based on differences in judgments (Firestone \& Scholl, in press-a; Wesp \& Gasper, 2012), the current data support our claim that the paddle effect is not judgment-based. The diverging outcomes across both experiments for the paddle effect (compared with the effect of trial outcome) rule out a judgment-based explanation.

\section{Postdiction as alternative hypothesis}

The claim that this data help rule out a judgment-based explanation of the paddle effect is predicated on the assertion that the effect of trial outcome is judgment-based. There is, however, an alternative explanation for the effect of trial outcome. In some cases, what happens after a visually presented event can influence the perceptual experience of the event. This is known as postdiction. Postdiction has been argued to be involved in the perception of the flash-lag effect (Eagleman \& Sejnowski, 2000), backward masking (Sheth et al., 2000), and perception of causality (Choi \& Scholl, 2006). According to a postdictive interpretation, the outcome of the trial as a miss or a hit would be integrated along with visual signals and would alter the perception of the speed of the ball. If the trial outcome effect was due to postdictive processes, instead of judgmentbased processes, the current data would then provide evidence that the paddle effect is not due to postdictive processes (as opposed to not being due to judgment-based processes). Thus, the interpretation of the trial outcome effect is critical for conclusions about the paddle effect.

We are unaware of how a postdictive account could be distinguished from a judgment-based account. One could run the Pong method but without a paddle and simply have the ball stop or continue on various trials. This, however, 
would not differentiate the two explanations because the ball's motion (stop vs. continue) could lead to judgment-based effects as well postdictive effects. We have several reasons to suspect that the trial outcome effect is due to judgments rather than postdiction. The effect of trial outcome is not robust. Judgment-based effects require that participants report on beliefs, despite being asked to report on perception, or that participants alter their judgments based on demand characteristics (e.g., the ball is supposed to look faster when missed so "fast" is the response). The prevalence by which these two events occur has not been systematically documented, but research on altering responses due to social pressure such as has been done by Asch (1955) reveals that participants conform approximately $29 \%$ of the time (Bond \& Smith, 1996). Trial outcome effects have thus far been found at a similar prevalence rate, which is consistent with the idea that the trial outcome effect is also judgment-based. If the effect of trial outcome were perceptual and due to postdictive processes, one might expect the effect to be more robust. In addition, these studies show differences in the trial outcome effect across age and across cover story and blocking success combinations. These differences are expected under a judgment-based explanation, whereas it is less clear how a postdictive account could explain the obtained patterns. Finally, the claim that trial outcome directly impacts perception of ball speed (via postdictive processes) is likely to be just as controversial as other claims related to action-specific effects. To date, there is little evidence to suggest this effect cannot be explained via other processes or pitfalls. Until evidence is presented to the contrary, it is reasonable to consider the effect of trial outcome to be judgment-based as opposed to a genuine perceptual effect. However, if one were to accept that trial outcome is due to postdictive processes, such a stance would support the general claims of the action-specific approach that a person's action can directly influence perceptual processes.

\section{Conclusion}

These data help to rule out the pitfalls related to judgmentbased effects. One of the strategies employed here was the exact strategy recommended by Firestone and Scholl (in press-a) when describing Pitfall 2 (perception vs. judgment). In addition, that a cover story did not eliminate the paddle effect also helps rule out Pitfall 3 (demand and response bias). In conjunction with previous studies that addressed the remaining pitfalls (Witt et al., in press), the data make a compelling case that this particular action-specific effect is perceptual. The implication is not that all action-specific effects are perceptual. Rather, the implication is that perception can be influenced by a perceiver's ability to act. As conceded by (Firestone \& Scholl, in press-b), the claim that perception is entirely immune to top-down influences can be felled with the demonstration of one effect that survives all pitfalls. With the current data, we have moved even closer to achieving this demonstration.

Authors' Note Jessica K. Witt and Nathan L. Tenhundfeld, Department of Psychology, Colorado State University, Fort Collins, CO 80523. Allison A. M. Bielak, Department of Human Development and Family Studies, Colorado State University, Fort Collins, CO 80523.

This work was supported by grants from the National Science Foundation to J.K.W. (BCS-1348916 and BCS-1632222).

\section{References}

Asch, S. E. (1955). Opinions and social pressure. Scientific American, 193(5), 31-35.

Balcetis, E., \& Dunning, D. (2010). Wishful seeing: More desired objects are seen as closer. Psychological Science, 21(1), 147-152. doi:10.1177/0956797609356283

Bhalla, M., \& Proffitt, D. R. (1999). Visual-motor recalibration in geographical slant perception. Journal of Experimental Psychology: Human Perception and Performance, 25(4), 1076-1096.

Bond, R., \& Smith, P. B. (1996). Culture and conformity: A meta-analysis of studies using Asch"s $(1952 \mathrm{~b}, 1956)$ line judgment task. Psychological Bulletin, 119(1), 111-137.

Choi, H., \& Scholl, B. J. (2006). Perceiving causality after the fact: Postdiction in the temporal dynamics of causal perception. Perception, 35(3), 385-399.

Cole, S., Balcetis, E., \& Dunning, D. (2013). Affective signals of threat produce perceived proximity. Psychological Science, 24, 34- 40.

Costello, M. C., Bloesch, E. K., Davoli, C. C., Panting, N. D., Abrams, R. A., \& Brockmole, J. R. (2015). Spatial representations in older adults are not modified by action: Evidence from tool use. Psychology and Aging, 30(3), 656-668. doi:10.1037/pag0000029

Durgin, F. H., Baird, J. A., Greenburg, M., Russell, R., Shaughnessy, K., \& Waymouth, S. (2009). Who is being deceived? The experimental demands of wearing a backpack. Psychonomic Bulletin \& Review, 16(5), 964-969. doi:10.3758/PBR.16.5.964

Eagleman, D. M., \& Sejnowski, T. J. (2000). Motion integration and postdiction in visual awareness. Science, 287, 2036-2038.

Fajen, B. R., \& Phillips, F. (2012). Spatial perception and action. In D. A. Waller \& L. Nadel (Eds.), Handbook of spatial cognition (pp. 6780). Washington, DC: American Psychological Association.

Firestone, C. (2013). How "paternalistic" is spatial perception? Why wearing a heavy backpack doesn't — and couldn't — make hills appear steeper. Perspectives on Psychological Science, 8(4), 455-473.

Firestone, C., \& Scholl, B. J. (2014). "Top-down" effects where none should be found: The El Greco fallacy in perception research. Psychological Science, 25(1), 38-46.

Firestone, C., \& Scholl, B. J. (in press-a). Cognition does not affect perception: Evaluating the evidence for 'top-down' effects. Behavioral and Brain Sciences.

Firestone, C., \& Scholl, B. J. (in press-b). Seeing and thinking: Foundational issues and empirical horizons [response to commentators]. Behavioral and Brain Sciences.

Foley, J. M. (1977). Effect of distance information and range on two indices of visually perceived distance. Perception, 6(4), 449-460.

Folstein, M., Folstein, S., \& McHugh, P. R. (1975). Mini-Mental State: A practical method for grading the cognitive state of patients for the clinician. Journal of Psychiatric Research, 12, 189-198.

Gescheider, G. A. (1997). Psychophysics: The fundamentals (3rd ed.). Mahwah, NJ: Erlbaum.

Gogel, W. C. (1990). A theory of phenomenal geometry and its applications. Perception \& Psychophysics, 48(2), 105-123. 
Gray, R. (2013). Being selective at the plate: Processing dependence between perceptual variables relates to hitting goals and performance. Journal of Experimental Psychology: Human Perception and Performance, 39(4), 1124-1142.

Gregory, R. L. (1997). Knowledge in perception and illusion. Philosophical Transactions of the Royal Society of London, Series B: Biological Sciences, 352, 1121-1127.

Helmholtz, H. v. (2000). Physiological optics (Vol III concerning the perceptions in general). In S. Yanstis (Ed.), Visual perception: Essential readings (pp. 24-44). Philadelphia, PA: Psychology Press (Original work published 1925).

Hess, T. M., Auman, C., Colcombe, S. J., \& Rahhal, T. A. (2003). The impact of stereotype threat on age differences in memory performance. Journal of Gerontology: Psychological Sciences, 58(1), 3 P11.

Hubbard, T. L. (2012). Visual perception of force: Comment on White (2012). Psychological Bulletin, 138(4), 616-623.

Hubbard, T. L. (2013). Phenomenal causality II: Integration and implication. Axiomathes, 23(3), 485-524.

Jacoby, L. L., \& Rhodes, M. G. (2006). False remembering in the aged. Current Directions in Psychological Science, 15(2), 49-53.

Levin, D. T., \& Banaji, M. R. (2006). Distortions in the perceived lightness of faces: The role of race categories. Journal of Experimental Psychology: General, 135, 501-512.

Linkenauger, S. A., Bulthoff, H. H., \& Mohler, B. J. (2015). Virtual arm's reach influences perceived distance but only after experience reaching. Neuropsychologia, 70, 393-401. doi:10.1016/j. neuropsychologia.2014.10.034

Linkenauger, S. A., Leyrer, M., Buelthoff, H. H., \& Mohler, B. J. (2013). Welcome to wonderland: The influence of the size and shape of a virtual hand on the perceived size and shape of virtual objects. PLOS ONE, 8(7), e68594.

Loomis, J. M., \& Philbeck, J. W. (2008). Measuring perception with spatial updating and action. In R. L. Klatzky, M. Behrmann, \& B. MacWhinney (Eds.), Embodiment, ego-space, and action (pp. 144). Mahwah, NJ: Erlbaum.

Norman, J. F., Cheeseman, J. R., Baxter, M. W., Thomason, K. E., Adkins, O. C., \& Rogers, C. E. (2014). Aging and visual length discrimination: Sequential dependencies, biases, and the effects of multiple implicit standards. Vision Research, 98, 89-98.

Orne, M. T. (1962). On the social psychology of the psychological experiment: With particular reference to demand characteristics and their implications. American Psychologist, 17(11), 776-783.

Philbeck, J. W., \& Loomis, J. M. (1997). Comparison of two indicators of perceived egocentric distance under full-cue and reduced-cue conditions. Journal of Experimental Psychology: Human Perception and Performance, 23(1), 72-85.

Philbeck, J. W., \& Witt, J. K. (2015). Action-specific influences on perception and post-perceptual processes: Present controversies and future directions. Psychological Bulletin, 141(6), 1120-1144. doi:10.1037/a0039738

Ramachandran, V. S. (1988). Perception of shape from shading. Nature, $331,163-166$.

Rock, I. (1983). The logic of perception. Cambridge, MA: MIT Press.

Scholl, B. J., \& Gao, T. (2013). Perceiving animacy and intentionality: Visual processing or higher-level judgment? In M. D. Rutherford \& V. A. Kuhlmeier (Eds.), Social perception: Detection and interpretation of animacy, agency, and intention. Cambridge, MA: MIT Press.

Sekuler, R., Sekuler, A. B., \& Lau, R. (1997). Sound alters visual motion perception. Nature, 385, 308.

Shaffer, D. M., McManama, E., Swank, C., \& Durgin, F. H. (2013). Sugar and space? Not the case: Effects of low blood glucose on slant estimation are mediated by beliefs. i-Perception, 4, 147-155.

Shams, L., Kamitani, Y., \& Shimojo, S. (2000). What you see is what you hear. Nature, 408, 788
Sheth, B. R., Nijhawan, R., \& Shimojo, S. (2000). Changing objects lead briefly flashed ones. Nature Neuroscience, 3(5), 489-495.

Stefanucci, J. K., \& Proffitt, D. R. (2009). The roles of altitude and fear in the perception of height. Journal of Experimental Psychology: Human Perception and Performance, 35(2), 424-438. doi:10.1037/ a0013894

Stefanucci, J. K., Proffitt, D. R., Clore, G., \& Parekh, N. (2008). Skating down a steeper slope: Fear influences the perception of geographical slant. Perception, 37, 321-323.

Stocker, A. A., \& Simoncelli, E. P. (2006). Noise characteristics and prior expectations in human visual speed perception. Nature Neuroscience, 9, 578-585.

Sugovic, M., Turk, P., \& Witt, J. K. (2016). Perceived distance and obesity: It's what you weigh, not what you think. Acta Psychologica, $165,1-8$.

Taylor, J. E. T., Witt, J. K., \& Sugovic, M. (2011). When walls are no longer barriers: Perception of wall height in parkour. Perception, 40(6), 757-760. doi:10.1068/P6855

Taylor-Covill, G. A. H., \& Eves, F. F. (2013). Slant perception for stairs and screens: Effects of sex and fatigue in a laboratory environment. Perception, 42(4), 459-469.

Taylor-Covill, G. A. H., \& Eves, F. F. (2014). When what we need influences what we see: Choice of energetic replenishment is linked with perceived steepness. Journal of Experimental Psychology: Human Perception and Performance, 40(3), 915-919.

Taylor-Covill, G. A. H., \& Eves, F. F. (2016). Carrying a biological "backpack": Quasi-experimental effects of weight status and body fat change on perceived steepness. Journal of Experimental Psychology: Human Perception and Performance, 42(3), 331-338.

Wang, M., \& Chen, Y. (2004). Age differences in the correction processes of context-induced biases: When correction succeeds. Psychology and Aging, 19(3), 536-540.

Wesp, R., Cichello, P., Gracia, E. B., \& Davis, K. (2004). Observing and engaging in purposeful actions with objects influences estimates of their size. Perception \& Psychophysics, 66(8), 1261-1267.

Wesp, R., \& Gasper, J. (2012). Is size misperception of targets simply justification for poor performance? Perception, 41(8), 994-996. doi:10.1068/p7281

White, P. A. (2012). The experience of force: The role of haptic experience of forces in visual perception of object motion and interactions, mental simulation, and motion-related judgments. Psychological Bulletin, 138, 589-615.

Witt, J. K. (2011). Action's effect on perception. Current Directions in Psychological Science, 20(3), 201-206. doi:10.1177/ 0963721411408770

Witt, J. K. (in press). Spatial biases from action. In T. Hubbard (Ed.), Spatial biases. Cambridge, UK: Cambridge University Press.

Witt, J. K., \& Proffitt, D. R. (2005). See the ball, hit the ball-Apparent ball size is correlated with batting average. Psychological Science, 16(12), 937-938. doi:10.1111/j.1467-9280.2005.01640.x

Witt, J. K., Proffitt, D. R., \& Epstein, W. (2005). Tool use affects perceived distance, but only when you intend to use it. Journal of Experimental Psychology: Human Perception and Performance, 31(5), 880-888. doi:10.1037/0096-1523.31.5.880

Witt, J. K., South, S. C., \& Sugovic, M. (2014). A perceiver's own abilities influence perception, even when observing others. Psychonomic Bulletin \& Review, 21(2), 384-389.

Witt, J. K., \& Sugovic, M. (2010). Performance and ease influence perceived speed. Perception, 39(10), 1341-1353. doi:10.1068/P6699

Witt, J. K., \& Sugovic, M. (2012). Does ease to block a ball affect perceived ball speed? Examination of alternative hypotheses. Journal of Experimental Psychology: Human Perception and Performance, 38(5), 1202-1214. doi:10.1037/a0026512

Witt, J. K., \& Sugovic, M. (2013a). Catching ease influences perceived speed: Evidence for action-specific effects from action-based measures. Psychonomic Bulletin \& Review, 20, 1364-1370. 
Witt, J. K., \& Sugovic, M. (2013b). Response bias cannot explain actionspecific effects: Evidence from compliant and non-compliant participants. Perception, 42, 138-152.

Witt, J. K., Sugovic, M., Tenhundfeld, N. T., \& King, Z. R. (in press). An action-specific effect on perception that avoids all pitfalls. Behavioral and Brain Sciences.

Witt, J. K., Sugovic, M., \& Dodd, M. D. (2016). Action-specific perception of speed is independent of attention. Attention, Perception, \& Psychophysics, 78(3), 880-890. doi:10.3758/s13414-015-1047-6
Witt, J. K., Sugovic, M., \& Taylor, J. E. T. (2012). Action-specific effects in a social context: Others' abilities influence perceived speed. Journal of Experimental Psychology: Human Perception and Performance, 38(3), 715-725. doi:10.1037/a0026261

Woods, A. J., Philbeck, J. W., \& Danoff, J. V. (2009). The various perceptions of distance: An alternative view of how effort affects distance judgments. Journal of Experimental Psychology: Human Perception and Performance, 35(4), 1104-1117. doi:10.1037/ a0013622 